Moroccan J. of Pure and Appl. Anal. (MJPAA)

Volume 6(2), 2020, Pages 168-183

ISSN: Online 2351-8227 - Print 2605-6364

DOI: 10.2478/mjpaa-2020-0013

\title{
A generalized sequential problem of Lane-Emden type via fractional calculus
}

\author{
YAZID GOUARI ${ }^{1}$, ZoubIR DAHMANI ${ }^{2}$, AND AMETH NDIAYE ${ }^{3}$
}

Aвstract. In this paper, we combine the Riemann-Liouville integral operator and Caputo derivative to investigate a nonlinear time-singular differential equation of Lane Emden type. The considered problem involves $n$ fractional Caputo derivatives under the conditions that neither commutativity nor semi group property is satisfied for these derivatives. We prove an existence and uniqueness analytic result by application of Banach contraction principle. Then, another result that deals with the existence of at least one solution is delivered and some sufficient conditions related to this result are established by means of the fixed point theorem of Schaefer. We end the paper by presenting to the reader some illustrative examples.

Mathematics Subject Classification (2010). 30C45, 39B72, 39B82.

Key words and phrases. Caputo derivative, Lane-Emden equation, Existence of solution, Eixed point, Singular differential equation.

\section{Introduction}

Fractional calculus and differential equations of fractional order are of great importance since they can be used in analyzing and modeling real word phenomena [10,11, 21]. Recently, there has been a very important progress in the study of the theory of differential equations of fractional order. For some related research works with applications, the reader can consult the references $[3,4,20,22]$. In particular, research on the existence of unique solutions for fractional

Received 15 May 2020 - Accepted: 10 July 2020 .

(C) This article is published with open access by Sidi Mohamed Ben Abdallah University.

The Author(s): ${ }^{1,2}$ LPAM, Faculty of SEI, UMAB University of Mostaganem, Mostaganem, Algeria. e-mail: ${ }^{1}$ gouariyazid@gmail.com

e-mail: ${ }^{2} z$ dahmani@yahoo.fr (Corresponding author).

${ }^{3}$ Department of Mathematics, FASTEF, Université Cheikh Anta Diop, Dakar, Senegal.

e-mail: ${ }^{3}$ ameth1.ndiaye@ucad.edu.sn . 
differential equations is of big importance since it help physicians to better understand the behaviour of real word phenomena. For more details, see the papers $[2,15,20,22,23,24,27]$. Moreover, the singular differential equations are also very important in applied sciences, for more information, see $[2,6,7,25]$. Among these equations, we cite the standard Lane-Emden equation which is part of the present work. This equation has a considerable importance in astrophysics, for more details, one can consult [19, 26, 27] and the reference therein. Before recalling some other equations and problems that have motivated the present work, we remind the reader that the standard form of Lane Emden equation has the following form:

$$
y^{\prime \prime}(t)+\frac{a}{t} y^{\prime}(t)+f(t, y(t))=g(t), t \in(0,1], a \geq 0,
$$

with

$$
y(0)=a_{1}, y^{\prime}(0)=a_{2},
$$

where $a_{1}, a_{2}$ are constants, $f$ and $g$ are continuous functions (see [28]).

In [23], the authors have proposed the following fractional order Lane Emden model:

$$
\left\{\begin{array}{c}
D^{\alpha} y(t)+\frac{k}{t^{\alpha-\beta}} D^{\beta} y(t)+f(t, y(t))=g(t), t \in(0,1], \\
k \geq 0,1<\alpha \leq 2,0<\beta \leq 1,
\end{array}\right.
$$

with

$$
y(0)=y_{0}, y^{\prime}(0)=y_{1},
$$

where $y_{0}$ and $y_{1}$ are constants, $f$ and $g$ are two continuous functions and the derivatives are in the sense of Riemann-Liouville. The authors have used the collocation method to establish some numerical solutions for this model.

In $[18,19]$, Rabha W. Ibrahim has studied two sequential singular equations having the same form, but different in their initial conditions. The considered equations are given by:

$$
\left\{\begin{array}{c}
D^{\beta}\left(D^{\alpha}+\frac{a}{t}\right) u(t)+f(t, u(t))=g(t), \\
0<\alpha, \beta \leq 1,0<t \leq 1, a \geq 0 .
\end{array}\right.
$$

For her first sequential singular equation, Rabha W. Ibrahim has taken the conditions $u(0)=$ $u(1)=u(r)=0,0<r<1$; for this case, the existence of solutions by Krasnoselskii theorem has been studied in [18]. The second sequential problem that has been considered by Rahba $\mathrm{W}$. Ibrahim has the conditions $u(0)=\mu, u(1)=v$; for this case, the existence and the Ulam stability of solutions have been discussed in [19].

Also in [8], A. Bekkouche et al. have studied the existence of solutions and the $\Delta$-Ulam stabilities for the following two dimension non homogeneous Lane-Emden fractional system:

$$
\left\{\begin{array}{c}
D^{\beta_{1}}\left(D^{\alpha_{1}}+b_{1} g_{1}(t)\right) x_{1}(t)+f_{1}\left(t, x_{1}(t), x_{2}(t)\right) \\
=\omega_{1} S_{1}\left(t, x_{1}(t), x_{2}(t)\right), 0<t<1, \\
D^{\beta_{2}}\left(D^{\alpha_{2}}+b_{2} g_{2}(t)\right) x_{2}(t)+f_{2}\left(t, x_{1}(t), x_{2}(t)\right) \\
=\omega_{2} S_{2}\left(t, x_{1}(t), x_{2}(t)\right), 0<t<1 \\
x_{k}(0)=0, D^{\alpha} x_{k}(1)+b_{k} g_{k}(1) x_{k}(1)=0
\end{array}\right.
$$


under the conditions: $0<\beta_{k}<1,0<\alpha_{k}<1, b_{k} \geq 0,0<\omega_{k}<\infty, k=1,2$ and the derivatives $D^{\beta_{k}}$ and $D^{\alpha_{k}}$ are in the sense of Caputo. The functions $f_{k}:[0,1] \times \mathbb{R}^{2} \rightarrow \mathbb{R}$ and $S_{k}:[0,1] \times$ $\mathbb{R}^{2} \rightarrow \mathbb{R}$ are continuous, $\left.\left.g_{k}:\right] 0,1\right] \rightarrow[0,+\infty)$ is continuous and singular at $t=0$.

In the paper [9], A. Benzidane and Z. Dahmani have considered the following class of nonlinear equations of Lane Emden type:

$$
\left\{\begin{array}{l}
D^{\beta_{1}}\left(D^{\alpha_{1}}+g_{1}(t)\right) x_{1}(t)+f_{1}\left(t, x_{1}(t), x_{2}(t), D^{\delta_{1}} x_{1}(t), D^{\delta_{2}} x_{2}(t)\right) \\
\quad=h_{1}\left(t, x_{1}(t), x_{2}(t)\right) \\
D^{\beta_{2}}\left(D^{\alpha_{2}}+g_{2}(t)\right) x_{2}(t)+f_{2}\left(t, x_{1}(t), x_{2}(t), D^{\delta_{1}} x_{1}(t), D^{\delta_{2}} x_{2}(t)\right) \\
=h_{2}\left(t, x_{1}(t), x_{2}(t)\right) \\
x_{k}(0)=a_{k}, x_{k}(1)=b_{k}, t \in J
\end{array}\right.
$$

where $J=[0,1], 0<\alpha_{k}, \beta_{k}<1,0<\delta_{k}<\alpha_{k}<1, k=1$, 2; the functions $f_{k}:[0,1] \times \mathbb{R}^{4}$, $k=1,2$ are continuous, $g_{k}:(0,1] \longrightarrow[0,+\infty)$ are continuous functions, singular at $t=0$, and $\lim _{t \rightarrow 0^{+}} g_{k}(t)=\infty$; the operators $D^{\alpha_{k}}, D^{\beta_{k}}$ and $D^{\delta_{k}} k=1,2$ are the derivatives in the sense of Caputo and the constants $a_{k}, b_{k}$ are reals. The authors have studied the existence and uniqueness of solutions and the Ulam stability for the considered class.

Recently, Y. Bahous and Z. Dahmani [5] have considered a Lane Emden type problem involving both Caputo derivative and Riemann-Liouville integral in its nonlinearities. The problem is the following:

$$
\left\{\begin{array}{l}
D^{\beta}\left(D^{\alpha}+\frac{k}{t^{\lambda}}\right) y(t)+f\left(t, y(t), D^{\delta} y(t)\right)+g\left(t, y(t), I^{\rho} y(t)\right) \\
=h(t), t \in] 0,1[ \\
y(0)=v, y(1)=b \int_{0}^{\eta} q(s) y(s) d s, 0<\eta<1 \\
0<\beta, \alpha<1, k>0, \lambda>0
\end{array}\right.
$$

where $D^{\alpha}$ is in the sense of Caputo, $I^{\rho}$ is the Riemann-Liouville integral of order $\rho$, the functions $f, g:[0,1] \times \mathbb{R}^{2} \longrightarrow \mathbb{R}$ are continuous, and $h$ and $q$ are continuous over $[0,1]$. The authors have investigated the existence and uniqueness of solutions. Then, they have studied the Ulam-Hyers stability.

Very recently, Y. Gouari et al. [16] have proposed the following nonlinear singular integrodifferential equation of Lane Emden type with nonlocal multi point integral conditions:

$$
\left\{\begin{array}{l}
D^{\beta}\left(D^{\alpha}+\frac{k}{t^{\lambda}}\right) y(t)+\Delta_{1} f\left(t, y(t), D^{\delta} y(t)\right)+\Delta_{2} g\left(t, y(t), I^{\rho} y(t)\right) \\
+h(t, y(t))=l(t) \\
y(0)=0 \\
y(1)=b \int_{0}^{\eta} y(s) d s, 0<\eta<1 \\
I^{q} y(u)=y(1), 0<u<1 \\
k>0,0<\lambda \leq 1,1 \leq \beta \leq 2,0 \leq \alpha, \delta \leq 1
\end{array}\right.
$$


where $\Delta_{1}>0, \Delta_{2}>0$ are positive real numbers, $I^{\rho}$ is the Riemann-Liouville integral of order $\rho$, and $f, g$ are two given functions defined on $[0,1] \times \mathbb{R}^{2}$, and $h$ and $l$ are two given functions defined over $[0,1]$. The authors have proved the existence and the uniqueness of solutions by application of Banach contraction principle, then, by means of Schaefer fixed point theorem, they have studied the existence of at least one solution for the problem.

In this paper, we are concerned with the following sequential time-singular fractional problem of Lane Emden type:

$$
\left\{\begin{array}{l}
D^{\alpha_{1}}\left(D^{\alpha_{2}} \ldots\left(D^{\alpha_{n}}\left(D^{\beta}+\frac{k}{t^{\lambda}}\right)\right) \ldots\right) u(t)+f\left(t, u(t), D^{\delta} u(t)\right)+g\left(t, u(t), I^{\rho} u(t)\right) \\
+h(t, u(t))=l(t), \quad t \in] 0,1[, \\
u(0)=0 \\
u(1)=\theta \\
D^{\alpha_{n}}\left(D^{\beta} u(0)\right)=0, \\
D^{\alpha_{n-1}}\left(D^{\alpha_{n}}\left(D^{\beta} u(0)\right)\right)=0, \\
\vdots \\
D^{\alpha_{3}}\left(D^{\alpha_{4}} \ldots\left(D^{\alpha_{n}}\left(D^{\beta} u(0)\right)\right) \ldots\right)=0, \\
D^{\alpha_{2}}\left(D^{\alpha_{3}} \ldots\left(D^{\alpha_{n}}\left(D^{\beta} u(1)+\phi_{k, \lambda}(1) u(1)\right)\right) \ldots\right)=0, \\
k>0 .
\end{array}\right.
$$

For (1.8), we take $J:=[0,1], 0 \leq \beta \leq 1,0 \leq \alpha_{i}<1 ; i=1,2, \ldots, n, \delta<\min \left(\beta, \alpha_{i}\right), \phi_{k, \lambda}(t)=\frac{k}{t^{\lambda}}$, the sequential derivatives are in the sense of Caputo, $I^{\rho}$ denotes the Riemann-Liouville integral of order $\rho$, and $f, g: J \times \mathbb{R}^{2} \rightarrow \mathbb{R}$ are two given functions, also $h: J \times \mathbb{R} \rightarrow \mathbb{R}$ is a given function and $l$ is a function which is defined on $J$.

We need also to shed light on the fact that for the above problem, we take into account the following points:

1. We introduce the Caputo derivative in both sides of equation of the problem.

2. Also, we introduce the Riemann Liouville integral in one nonlinearity of the right hand side of the equation.

3. Another particularity of the problem, is that it includes $n$ sequential Caputo derivations which allow us to introduce a new sequential problem with absence of commutativity and semi group properties between the introduced derivatives; this absence is not true in the classical case of differentiation. (It is also absent when dealing with, at least, two parameters $\alpha_{1}, \alpha_{2} \in$ $[0,1]$, that satisfy $\left.\alpha_{1}+\alpha_{2} \notin[0,1]\right)$. So, for our case, we have to find new arguments to overcome this type of problem.

4. Another important point in this paper is the time singularity at the origin for the above problem.

So based on the above four conditions, we think that in this paper, we are concerned with a more general sequential problem of Lane Emden type; it is "more general" in the sense that it can be used to describe many problems that arise in mathematical physics, since it includes several particular types of equations with important applications. For instance, it is easy to verify that our problem includes the standard Lane Emden equation as a special case. Also, 
it includes the Emden Fowler equation that has been introduced to model several phenomena in mathematical physics and astrophysics, such as the theory of stellar structure. Also, the fractional problem proposed by the authors of [16] can be derived from the above problem under some special values on the parameters and the functions of our problem.

To the best of our knowledge, this is the first time in the literature where such problem is investigated.

The paper is organised as follows: in section 2, we recall some definitions and lemmas that are used for the proof of our main results. In section 3, we prove the main theorems of this paper, and we discuss some illustrative examples. In the last section, a conclusion follows.

\section{Preliminaries}

In this paper, we work with the following definitions and lemmas. We refer the reader to [24] for more information.

Definition 2.1. The Riemann-Liouville integral of order $\alpha>0$ for all continuous function on $[0,1]$ is given by:

$$
I^{\alpha} f(t)=\frac{1}{\Gamma(\alpha)} \int_{0}^{t}(t-\tau)^{\alpha-1} f(\tau) d \tau,
$$

with $\Gamma(\alpha):=\int_{0}^{\infty} e^{-u} u^{\alpha-1} d u$.

Definition 2.2. If $f \in C^{n}([0,1], \mathbb{R})$ and $n-1<\alpha \leq n$, then, the Caputo derivative is given by:

$$
\begin{aligned}
D^{\alpha} f(t) & =I^{n-\alpha} \frac{d^{n}}{d t^{n}}(f(t)) \\
& =\frac{1}{\Gamma(n-\alpha)} \int_{0}^{t}(t-s)^{n-\alpha-1} f^{(n)}(s) d s .
\end{aligned}
$$

Lemma 2.1. Taking $n \in \mathbb{N}^{*}$ and $n-1<\alpha<n$, then the solutions of $D^{\alpha} y(t)=0$ are:

$$
y(t)=\sum_{i=0}^{n-1} c_{i} t^{i}
$$

$c_{i} \in \mathbb{R}, i=0,1,2, . ., n-1$.

Lemma 2.2. Taking $n \in \mathbb{N}^{*}, n-1<\alpha<n$, then,

$$
I^{\alpha} D^{\alpha} y(t)=y(t)+\sum_{i=0}^{n-1} c_{i} t^{i},
$$

and $c_{i} \in \mathbb{R}, i=0,1,2, . ., n-1$.

The following result establishes a relation between the integral representation and the differential problem given in (1.8). 
Lemma 2.3. Let $G \in C([0,1])$. Then, we can state that

$$
\left\{\begin{array}{c}
\left.D^{\alpha_{1}}\left(D^{\alpha_{2}} \ldots\left(D^{\alpha_{n}}\left(D^{\beta}+\frac{k}{t^{\lambda}}\right)\right) \ldots\right) u(t)=G(t), t \in\right] 0,1[, \\
u(0)=0, \\
u(1)=\theta, \\
D^{\alpha_{n}}\left(D^{\beta} u(0)\right)=0, \\
D^{\alpha_{n-1}}\left(D^{\alpha_{n}}\left(D^{\beta} u(0)\right)\right)=0, \\
\vdots \\
D^{\alpha_{3}}\left(D^{\alpha_{4}} \ldots\left(D^{\alpha_{n}}\left(D^{\beta} u(0)\right)\right) \ldots\right)=0, \\
D^{\alpha_{2}}\left(D^{\alpha_{3}} \ldots\left(D^{\alpha_{n}}\left(D^{\beta} u(1)+\phi_{k, \lambda}(1) u(1)\right)\right) \ldots\right)=0, \\
k>0,0 \leq \alpha_{i}<1,0 \leq \beta \leq 1 ; i=1,2, \ldots, n
\end{array}\right.
$$

admits as integral solution the following expression

$$
\begin{aligned}
u(t)= & \frac{1}{\Gamma(\beta)} \int_{0}^{t}(t-s)^{\beta-1}\left(\frac{1}{\Gamma\left(\sum_{i=1}^{n} \alpha_{i}\right)} \int_{0}^{s}(s-\tau)^{\sum^{i=1} \alpha_{i}-1} G(\tau) d \tau-\frac{k}{s^{\lambda}} u(s)\right) d s \\
& -\frac{\int_{0}^{1}(1-s)^{\alpha_{1}-1} G(s) d s}{\Gamma\left(\alpha_{1}\right) \Gamma\left(\sum_{i=2}^{n} \alpha_{i}+\beta+1\right)} t^{n=2} \alpha_{i}+\left[\frac{\int_{0}^{1}(1-s)^{\alpha_{1}-1} G(s) d s}{\Gamma\left(\alpha_{1}\right) \Gamma\left(\sum_{i=2}^{n} \alpha_{i}+\beta+1\right)}\right] t^{\beta} \\
& -\left[\frac{1}{\Gamma(\beta)} \int_{0}^{1}(1-s)^{\beta-1}\left(\frac{1}{\Gamma\left(\sum_{i=1}^{n} \alpha_{i}\right)} \int_{0}^{s}(s-\tau)^{i=1} \sum_{i}^{n} G(\tau) d \tau-\frac{k}{s^{\lambda}} u(s)\right) d s+\theta\right] t^{\beta} .
\end{aligned}
$$

Proof. We use the property established in Lemma 2.2 to (2.5), we find that

$$
\begin{array}{r}
u(t)=I^{\beta}\left(I^{\sum_{i=1}^{n} \alpha_{i}} G(s)-\frac{k}{s^{\lambda}}\right)(t)+\frac{c_{0}}{\Gamma\left(\sum_{i=2}^{n} \alpha_{i}+\beta+1\right)} t^{\sum^{i=2} \alpha_{i}+\beta}+\frac{c_{1}}{\Gamma\left(\sum_{i=3}^{n} \alpha_{i}+\beta+1\right)} t^{i=3} \alpha_{i}^{n}+\beta \\
+\frac{c_{2}}{\Gamma\left(\sum_{i=4}^{n} \alpha_{i}+\beta+1\right)} t^{i=4} \alpha_{i}+\ldots+\frac{c_{n-2}}{\Gamma\left(\alpha_{n}+\beta+1\right)} t^{\alpha_{n}+\beta}+\frac{c_{n-1}}{\Gamma(\beta+1)} t^{\beta}+c_{n} .
\end{array}
$$

Some of the initial conditions allow us to write:

$$
\begin{gathered}
u(0)=0 \Rightarrow c_{n}=0 \\
D^{\alpha_{2}}\left(D^{\alpha_{3}} \ldots\left(D^{\alpha_{n}}\left(D^{\beta} u(1)+\phi_{k, \lambda}(1) u(1)\right)\right) \ldots\right)=0 \Rightarrow c_{0}=-I^{\alpha_{1}} G(1) \\
D^{\alpha_{n}}\left(D^{\beta} u(0)\right)=0 \Rightarrow c_{n-2}=0 \\
D^{\alpha_{n-1}}\left(D^{\alpha_{n}}\left(D^{\beta} u(0)\right)\right)=0 \Rightarrow c_{n-3}=0
\end{gathered}
$$




$$
\begin{aligned}
& \begin{array}{c}
\vdots \\
D^{\alpha_{4}}\left(D^{\alpha_{5}} \ldots\left(D^{\alpha_{n}}\left(D^{\beta} u(0)\right)\right) \ldots\right)=0 \Rightarrow c_{2}=0
\end{array} \\
& D^{\alpha_{3}}\left(D^{\alpha_{4}} \ldots\left(D^{\alpha_{n}}\left(D^{\beta} u(0)\right)\right) \ldots\right)=0 \Rightarrow c_{1}=0 \\
& u(1)=\theta \Rightarrow \\
& \frac{c_{n-1}}{\Gamma(\beta+1)}=\frac{\int_{0}^{1}(1-s)^{\alpha_{1}-1} G(s) d s}{\Gamma\left(\alpha_{1}\right) \Gamma\left(\sum_{i=2}^{n} \alpha_{i}+\beta+1\right)} \\
& -\frac{1}{\Gamma(\beta)} \int_{0}^{1}(1-s)^{\beta-1}\left(\frac{1}{\Gamma\left(\sum_{i=1}^{n} \alpha_{i}\right)} \int_{0}^{s}(s-\tau)^{\sum_{i=1}^{n} \alpha_{i}-1} G(\tau) d \tau-\frac{k}{s^{\lambda}} u(s)\right) d s+\theta .
\end{aligned}
$$

Replacing $c_{0}, c_{1}, c_{2}, \ldots, c_{n}$ in (2.7), we end the proof.

Let us now transforming the above problem to a fixed point one. We begin by considering the following notions related to Banach spaces:

and

$$
\begin{gathered}
X:=\left\{x \in C(J, \mathbb{R}), D^{\delta} x \in C(J, \mathbb{R})\right\} ; \\
\|x\|_{X}=\operatorname{Max}\left\{\|x\|_{\infty},\left\|D^{\delta} x\right\|_{\infty}\right\},
\end{gathered}
$$

$$
\|x\|_{\infty}=\sup _{t \in J}|x(t)|,\left\|D^{\delta} x\right\|_{\infty}=\sup _{t \in J}\left|D^{\delta} x(t)\right| .
$$

Then, we consider the nonlinear operator $H: X \rightarrow X$ that takes the form:

$$
\begin{aligned}
H u(t)= & \frac{1}{\Gamma(\beta)} \int_{0}^{t}(t-s)^{\beta-1}\left[\frac { 1 } { \Gamma ( \sum _ { i = 1 } ^ { n } \alpha _ { i } ) } \int _ { 0 } ^ { s } ( s - \tau ) ^ { \sum ^ { n } \alpha _ { i } - 1 } \left(l(\tau)-h(\tau, u(\tau))-f\left(\tau, u(\tau), D^{\delta} u(\tau)\right)\right.\right. \\
& \left.\left.-g\left(\tau, u(\tau), I^{\rho} u(\tau)\right)\right) d \tau-\frac{k}{s^{\lambda}} u(s)\right] d s-\frac{\sum^{i=2} \alpha_{i}+\beta}{\Gamma\left(\alpha_{1}\right) \Gamma\left(\sum_{i=2}^{n} \alpha_{i}+\beta+1\right)} \int_{0}^{1}(1-s)^{\alpha_{1}-1}(l(s) \\
& \left.-h(s, u(s))-f\left(s, u(s), D^{\delta} u(s)\right)-g\left(s, u(s), I^{\rho} u(s)\right)\right) d s-\left[\frac { 1 } { \Gamma ( \beta ) } \int _ { 0 } ^ { 1 } ( 1 - s ) ^ { \beta - 1 } \left(\frac{1}{\Gamma\left(\sum_{i=1}^{n} \alpha_{i}\right)}\right.\right. \\
& \times \int_{0}^{s}(s-\tau)^{i=1} \alpha_{i}^{n}-1 \\
& \left.\left.-\frac{k}{s^{\lambda}} u(s)\right) d s+\theta\right] t^{\beta},
\end{aligned}
$$

such that $\lambda<1$.

At the end of this second section, we are able to prove the results. 


\section{Main Results}

First, we note that we need to work with the following hypotheses:

(A1) : The functions $f$ and $g$ are defined and continuous on $J \times \mathbb{R}^{2}$ and $h$ is defined and continuous on $J \times \mathbb{R}$.

(A2) : There are four nonnegative constants $L_{f 1}, L_{f 2}, L_{g 1}, L_{g 2}$; for any $\mathrm{t} \in J, x_{i}, x_{i}^{*} \in \mathbb{R}$,

$$
\begin{aligned}
& \left|f\left(t, x_{1}, x_{2}\right)-f\left(t, x_{1}{ }^{*}, x_{2}{ }^{*}\right)\right| \leq \sum_{i=1}^{2} L_{f i}\left|x_{i}-x_{i}{ }^{*}\right|, \\
& \left|g\left(t, x_{1}, x_{2}\right)-g\left(t, x_{1}{ }^{*}, x_{2}{ }^{*}\right)\right| \leq \sum_{i=1}^{2} L_{g i}\left|x_{i}-x_{i}{ }^{*}\right|,
\end{aligned}
$$

and there is one positive number $r_{0}$; for any $t \in J, x, y \in \mathbb{R}$,

$$
|h(t, x)-h(t, y)| \leq r_{0}|x-y| \text {. }
$$

We take

$$
L_{f}^{\prime}:=\operatorname{Max}\left(L_{f 1}, L_{f 2}\right), L_{g}^{\prime}:=\operatorname{Max}\left(L_{g 1}, L_{g 2}\right) .
$$

(A3) : There are three positive constants $M_{f}, M_{g}, M_{h}$; for any $t \in J, x \in \mathbb{R}^{2}, y \in \mathbb{R}$, we have

$$
|f(t, x)| \leq M_{f}, \quad|g(t, x)| \leq M_{g},|h(t, y)| \leq M_{h} .
$$

$(A 4)$ : We take: $\|l\|_{\infty}=M_{l}$.

Also, we consider the new expressions:

$$
\begin{aligned}
D 1= & 2\left[\left(r_{0}+2 L_{f}^{\prime}+L_{g}^{\prime}+\frac{L_{g}^{\prime}}{\Gamma(\rho+1)}\right)\left(\frac{1}{\Gamma\left(\sum_{i=1}^{n} \alpha_{i}+\beta+1\right)}+\frac{1}{\Gamma\left(\sum_{i=2}^{n} \alpha_{i}+\beta+1\right) \Gamma\left(\alpha_{1}+1\right)}\right)\right. \\
& \left.+\frac{k \Gamma(1-\lambda)}{\Gamma(\beta-\lambda+1)}\right], \\
D 2= & \left(r_{0}+2 L_{f}^{\prime}+L_{g}^{\prime}+\frac{L_{g}^{\prime}}{\Gamma(\rho+1)}\right)\left(\frac{1}{\Gamma\left(\sum_{i=1}^{n} \alpha_{i}+\beta-\delta+1\right)}\right. \\
& +\frac{1}{\Gamma\left(\sum_{i=2}^{n} \alpha_{i}+\beta-\delta+1\right) \Gamma\left(\alpha_{1}+1\right)}+\frac{\Gamma(\beta+1)}{\Gamma\left(\sum_{i=2}^{n} \alpha_{i}+\beta+1\right) \Gamma(\beta-\delta+1) \Gamma\left(\alpha_{1}+1\right)} \\
& \left.+\frac{\Gamma(\beta+1)}{\Gamma\left(\sum_{i=1}^{n} \alpha_{i}+\beta+1\right) \Gamma(\beta-\delta+1)}\right)+k \Gamma(1-\lambda)\left(\frac{1}{\Gamma(\beta-\delta-\lambda+1)}+\frac{1}{\Gamma(\beta-\lambda+1)}\right) .
\end{aligned}
$$

The first main result deals with the existence of one and only one solution for (1.8). It is based on the application of Banach fixed point theorem for contraction mappings. 
Theorem 3.1. If the conditions $(A i)_{i=2,3,4}$ are satisfied and $D<1, D:=\max \{D 1, D 2\}$, then, the problem (1.8) has a unique solution on $\mathrm{J}$.

Proof. It is sufficient for us to prove that $H$ is a contraction mapping.

Let $(x, y) \in X^{2}$. Then, it is clear that

$$
\begin{aligned}
\|H y-H x\|_{\infty} \leq & 2\left[\left(r_{0}+2 L_{f}^{\prime}+L_{g}^{\prime}+\frac{L_{g}^{\prime}}{\Gamma(\rho+1)}\right)\left(\frac{1}{\Gamma\left(\sum_{i=1}^{n} \alpha_{i}+\beta+1\right)}+\frac{1}{\Gamma\left(\sum_{i=2}^{n} \alpha_{i}+\beta+1\right) \Gamma\left(\alpha_{1}+1\right)}\right)\right. \\
& \left.+\frac{k \Gamma(1-\lambda)}{\Gamma(\beta-\lambda+1)}\right]\|y-x\|_{X} .
\end{aligned}
$$

On the other hand, since

$$
\begin{aligned}
D^{\delta} H u(t)= & \frac{1}{\Gamma(\beta-\delta)} \int_{0}^{t}(t-s)^{\beta-\delta-1}\left[\frac{1}{\Gamma\left(\sum_{i=1}^{n} \alpha_{i}\right)} \int_{0}^{s}(s-\tau)^{i=1} \alpha_{i}^{n}(l(\tau)-h(\tau, u(\tau))\right. \\
& \left.\left.-f\left(\tau, u(\tau), D^{\delta} u(\tau)\right)-g\left(\tau, u(\tau), I^{\rho} u(\tau)\right)\right) d \tau-\frac{k}{s^{\lambda}} u(s)\right] d s-\frac{\sum_{t^{i=2}}^{n} \alpha_{i}+\beta-\delta}{\Gamma\left(\alpha_{1}\right) \Gamma\left(\sum_{i=2}^{n} \alpha_{i}+\beta-\delta+1\right)} \\
& \times \int_{0}^{1}(1-s)^{\alpha_{1}-1}\left(l(s)-h(s, u(s))-f\left(s, u(s), D^{\delta} u(s)\right)-g\left(s, u(s), I^{\rho} u(s)\right)\right) d s \\
& +\frac{\Gamma\left(\alpha_{1}\right) \Gamma\left(\sum_{i=2}^{n} \alpha_{i}+\beta+1\right) \Gamma(\beta-\delta+1)}{\Gamma+1) t^{\beta-\delta}}(1-s)^{\alpha_{1}-1}(l(s)-h(s, u(s)) \\
& \left.-f\left(s, u(s), D^{\delta} u(s)\right)-g\left(s, u(s), I^{\rho} u(s)\right)\right) d s-\left[\frac { 1 } { \Gamma ( \beta ) } \int _ { 0 } ^ { 1 } ( 1 - s ) ^ { \beta - 1 } \left(\frac{1}{\Gamma\left(\sum_{i=1}^{n} \alpha_{i}\right)}\right.\right. \\
& \left.\left.\times \int_{0}^{s}(s-\tau)^{i=1} \sum_{k}^{n} u(s)\right) d s+\theta\right] \frac{\Gamma(\beta+1)}{\Gamma(\beta-\delta+1)} t^{\beta-\delta}, \\
& -\frac{\left(l(\tau)-h(\tau, u(\tau))-f\left(\tau, u(\tau), D^{\delta} u(\tau)\right)-g\left(\tau, u(\tau), I^{\rho} u(\tau)\right)\right) d \tau}{s^{\lambda}}
\end{aligned}
$$


then, with the same arguments as before, we have

$$
\begin{aligned}
\left\|D^{\delta} H y-D^{\delta} H x\right\|_{\infty} \leq & \left(r_{0}+2 L_{f}^{\prime}+L_{g}^{\prime}+\frac{L_{g}^{\prime}}{\Gamma(\rho+1)}\right)\left(\frac{1}{\Gamma\left(\sum_{i=1}^{n} \alpha_{i}+\beta-\delta+1\right)}\right. \\
& +\frac{1}{\Gamma\left(\sum_{i=2}^{n} \alpha_{i}+\beta-\delta+1\right) \Gamma\left(\alpha_{1}+1\right)}+\frac{\Gamma(\beta+1)}{\Gamma\left(\sum_{i=2}^{n} \alpha_{i}+\beta+1\right) \Gamma(\beta-\delta+1) \Gamma\left(\alpha_{1}+1\right)} \\
& \left.+\frac{\Gamma(\beta+1)}{\Gamma\left(\sum_{i=1}^{n} \alpha_{i}+\beta+1\right) \Gamma(\beta-\delta+1)}\right)\|y-x\|_{X} \\
& +k \Gamma(1-\lambda)\left(\frac{1}{\Gamma(\beta-\delta-\lambda+1)}+\frac{1}{\Gamma(\beta-\lambda+1)}\right)\|y-x\|_{X} .
\end{aligned}
$$

Thanks to (3.8) and (3.10), we obtain

$$
\|H y-H x\|_{X} \leq D\|x-y\|_{X}
$$

The proof is thus achieved.

The following main result deals with the existence of at least one solution of the studied problem.

Theorem 3.2. Under the hypotheses $(A 1),(A 3)$ and $(A 4)$, the problem (1.8) has at least one solution $u(t), t \in J$.

Proof. Let us prove the result by considering the following steps:

Continuous of $H$ : The proof is trivial and then it is omitted. (We just apply the fact that the functions are continuous).

Unifrom Boundness of $H$ : Let us take $r>0$ and consider the (bounded) ball $B_{r}:=$ $\left\{x \in X ;\|x\|_{X} \leq r\right\}$. For $y \in B_{r}$, in virtue of $(A 3)$ and $(A 4)$, we can write

$$
\begin{aligned}
\|H y\|_{\infty} \leq & 2\left[\left(M_{l}+M_{h}+M_{f}+M_{g}\right)\left(\frac{1}{\Gamma\left(\sum_{i=1}^{n} \alpha_{i}+\beta+1\right)}+\frac{1}{\Gamma\left(\sum_{i=2}^{n} \alpha_{i}+\beta+1\right) \Gamma\left(\alpha_{1}+1\right)}\right)\right. \\
& \left.+\frac{k \Gamma(1-\lambda)}{\Gamma(\beta-\lambda+1)}\right]+|\theta|<+\infty
\end{aligned}
$$


and

$$
\begin{aligned}
\left\|D^{\delta} H y\right\|_{\infty} \leq & \left(M_{l}+M_{h}+M_{f}+M_{g}\right)\left(\frac{1}{\Gamma\left(\sum_{i=1}^{n} \alpha_{i}+\beta-\delta+1\right)}\right. \\
& +\frac{1}{\Gamma\left(\sum_{i=2}^{n} \alpha_{i}+\beta-\delta+1\right) \Gamma\left(\alpha_{1}+1\right)}+\frac{\Gamma(\beta+1)}{\Gamma\left(\sum_{i=2}^{n} \alpha_{i}+\beta+1\right) \Gamma(\beta-\delta+1) \Gamma\left(\alpha_{1}+1\right)} \\
& \left.+\frac{\Gamma(\beta+1)}{\Gamma\left(\sum_{i=1}^{n} \alpha_{i}+\beta+1\right) \Gamma(\beta-\delta+1)}\right)+k \Gamma(1-\lambda)\left(\frac{1}{\Gamma(\beta-\delta-\lambda+1)}+\frac{1}{\Gamma(\beta-\lambda+1)}\right) \\
& +|\theta|<+\infty .
\end{aligned}
$$

The above two inequalities show that $\|H y\|_{X}<+\infty$.

Consequently $H$ is uniformly bounded.

Equicontinuity of $H$ : We prove that for any bounded set ( $B_{r}$ for instance), we obtain that $H\left(B_{r}\right)$ is an equicontinuous set of $X$.

Taking $t_{1}, t_{2} \in[0,1], t_{1}<t_{2}$ and consider the above (bounded) ball $B_{r}$ of $X$. So by considering $y \in B_{r}$, we can state that

$$
\begin{aligned}
\left|H y\left(t_{1}\right)-H y\left(t_{2}\right)\right| \leq & \frac{M_{l}+M_{h}+M_{f}+M_{g}}{\Gamma\left(\sum_{i=1}^{n} \alpha_{i}+\beta+1\right)} \mid t_{1} \alpha_{i=1}^{n} \alpha_{-t_{2}} \sum_{i=1}^{n} \alpha_{i}+\beta \\
& +k r \frac{\Gamma(1-\lambda)}{\Gamma(1-\lambda+\beta)}\left|t_{1}^{\beta-\lambda}-t_{2} \beta-\lambda\right| \\
& +\frac{M_{l}+M_{h}+M_{f}+M_{g}}{\Gamma\left(\sum_{i=2}^{n} \alpha_{i}+\beta+1\right) \Gamma\left(1+\alpha_{1}\right)}\left(\mid t_{1} \sum_{i=2}^{n} \alpha_{i}+\beta-t_{2}^{i=2} \alpha_{i}+\beta\right. \\
& \left.+\left(\frac{M_{l}+M_{h}+M_{f}+M_{g}}{\Gamma\left(\sum_{i=1}^{n} \alpha_{i}+\beta+1\right)}+k r \frac{\Gamma(1-\lambda)}{\Gamma(1-\lambda+\beta)}+\theta\right)\left|t_{1} \beta-t_{2} \beta\right|\right)
\end{aligned}
$$


and

$$
\begin{aligned}
& \left|D^{\delta} H y\left(t_{1}\right)-D^{\delta} H y\left(t_{2}\right)\right| \leq \frac{M_{l}+M_{h}+M_{f}+M_{g}}{\Gamma\left(\sum_{i=1}^{n} \alpha_{i}+\beta-\delta+1\right)}\left|t_{1}^{n} \alpha_{i}-\delta+\beta-t_{2} \sum_{i=1}^{n} \alpha_{i}-\delta+\beta\right| \\
& +k r \frac{\Gamma(1-\lambda)}{\Gamma(1-\lambda+\beta-\delta)}\left|t_{1}^{\beta-\delta-\lambda}-t_{2}^{\beta-\delta-\lambda}\right| \\
& +\frac{M_{l}+M_{h}+M_{f}+M_{g}}{\Gamma\left(\sum_{i=2}^{n} \alpha_{i}+\beta+1\right) \Gamma\left(1+\alpha_{1}\right)}\left|t_{1} \sum_{i=2}^{n} \alpha_{i}+\beta-\delta \sum_{t_{2}}^{n} \alpha_{i}+\beta-\delta\right| \\
& +\frac{\left(M_{l}+M_{h}+M_{f}+M_{g}\right) \Gamma(\beta+1)}{\Gamma\left(\sum_{i=2}^{n} \alpha_{i}+\beta+1\right) \Gamma(\beta-\delta+1) \Gamma\left(\alpha_{1}+1\right)}\left|t_{1}^{\beta-\delta}-t_{2}^{\beta-\delta}\right| \\
& +\left(\frac{M_{l}+M_{h}+M_{f}+M_{g}}{\Gamma\left(\sum_{i=1}^{n} \alpha_{i}+\beta+1\right)}+k r \frac{\Gamma(1-\lambda)}{\Gamma(1-\lambda+\beta)}+\theta\right) \frac{\Gamma(\beta+1)}{\Gamma(\beta-\delta+1)} \\
& \times\left|t_{1}{ }^{\beta-\delta}-t_{2}{ }^{\beta-\delta}\right| \text {. }
\end{aligned}
$$

For (3.13) and (3.14), their right hand sides tend to zero for $t_{1} \rightarrow t_{2}$.

As a consequence the Ascoli-Arzela theorem, we conclude that $H$ is completely continuous.

Boundness of $A_{\gamma}$ : The set $A_{\gamma}:=\{x \in X: x=\gamma H x, \gamma \in] 0,1[\}$ is bounded. Let $y \in A_{\gamma}$. Then we have $y=\gamma H y$ for some $0<\gamma<1$. Hence,

$$
\begin{aligned}
\|y\|_{\infty} \leq & \gamma\left(2 \left[\left(M_{l}+M_{h}+M_{f}+M_{g}\right)\left(\frac{1}{\Gamma\left(\sum_{i=1}^{n} \alpha_{i}+\beta+1\right)}+\frac{1}{\Gamma\left(\sum_{i=2}^{n} \alpha_{i}+\beta+1\right) \Gamma\left(\alpha_{1}+1\right)}\right)\right.\right. \\
& \left.\left.+\frac{k \Gamma(1-\lambda)}{\Gamma(\beta-\lambda+1)}\right]+|\theta|\right) .
\end{aligned}
$$


Do not forget that we have

$$
\begin{aligned}
\left\|D^{\delta} y\right\|_{\infty} \leq & \gamma\left(( M _ { l } + M _ { h } + M _ { f } + M _ { g } ) \left(\frac{1}{\Gamma\left(\sum_{i=1}^{n} \alpha_{i}+\beta-\delta+1\right)}\right.\right. \\
& +\frac{1}{\Gamma\left(\sum_{i=2}^{n} \alpha_{i}+\beta-\delta+1\right) \Gamma\left(\alpha_{1}+1\right)}+\frac{\Gamma(\beta+1)}{\Gamma\left(\sum_{i=2}^{n} \alpha_{i}+\beta+1\right) \Gamma(\beta-\delta+1) \Gamma\left(\alpha_{1}+1\right)} \\
& \left.+\frac{\Gamma(\beta+1)}{\Gamma\left(\sum_{i=1}^{n} \alpha_{i}+\beta+1\right) \Gamma(\beta-\delta+1)}\right)+k \Gamma(1-\lambda)\left(\frac{1}{\Gamma(\beta-\delta-\lambda+1)}+\frac{1}{\Gamma(\beta-\lambda+1)}\right) \\
& +|\theta|) .
\end{aligned}
$$

Using (3.11) and (3.12), we state that $\|y\|_{X}<\infty$. The set is thus bounded.

Consequently, thanks to Schaefer fixed point theorem, we deduce that $H$ has at least one fixed point. Thus, the problem (1.8) has a solution.

At the end of this section, let us give the following two examples.

\section{Example 3.1.}

$$
\left\{\begin{array}{l}
D^{0.1}\left(D^{0.4}\left(D^{0.3}\left(D^{0.2}\left(D^{0.9}+\frac{3}{10 t^{0.1}}\right)\right)\right) y(t)+\frac{|y(t)|}{(44 \pi+t)(1+|y(t)|)}+\frac{\left|D^{\delta} y(t)\right|}{(200+t)\left(1+\left|D^{\delta} y(t)\right|\right)}\right. \\
\left.+\frac{\cos \left(y(t)+I^{\rho} y(t)\right)}{400\left(t^{2}+1\right)}+\frac{|y(t)|}{\left(72 e^{t+2}\right)(1+|y(t)|)}=3 t, t \in\right] 0,1[ \\
y(0)=0 \\
y(1)=1 \\
D^{0.2}\left(D^{0.9} y(0)\right)=0 \\
D^{0.3}\left(D^{0.2}\left(D^{0.9} y(0)\right)\right)=0 \\
D^{0.4}\left(D^{0.3}\left(D^{0.2}\left(D^{0.9} y(1)+\phi_{\frac{3}{10}, 0.1}(1) y(1)\right)\right)=0,\right. \\
\phi_{\frac{3}{10}, 0.1}(t)=\frac{3}{10 t^{0.1}}
\end{array}\right.
$$

where: $f\left(t, x_{1}, x_{2}\right)=\frac{\left|x_{1}\right|}{(44 \pi+t)\left(1+\left|x_{1}\right|\right)}+\frac{\left|x_{2}\right|}{(200+t)\left(1+\left|x_{2}\right|\right)}, g\left(t, x_{1}, x_{2}\right)=\frac{\cos \left(x_{1}+x_{2}\right)}{400\left(t^{2}+1\right)}$,

$$
\begin{aligned}
& h(t, x)=\frac{|x|}{\left(72 e^{t+2}\right)(1+|x|)}, l(t)=3 t, \delta=0.1, \rho=0.5, D 1=0.9613, D 2=0.9924, \text { and } \\
& D=\max \{D 1, D 2\}=0.9924 .
\end{aligned}
$$

The conditions of our first main result hold. Therefore, the example has a unique solution on $[0,1]$. 


\section{Example 3.2.}

$$
\left\{\begin{array}{l}
D^{0.2}\left(D^{0.4}\left(D^{0.4}\left(D^{0.2}\left(D^{0.2}\left(D^{\frac{1}{2}}+\frac{2}{5 t^{0.2}}\right)\right)\right)\right) y(t)+\frac{\sin y(t)+\sin D^{\delta} y(t)}{100 e^{t^{2}+1}}+\right. \\
\left.+\frac{1}{48 e^{t}}\left(\cos y(t)+\frac{\left|I^{\rho} y(t)\right|}{1+\left|I^{\rho} y(t)\right|}\right)+\frac{|y(t)|}{\left(144 e^{t^{2}}\right)(1+|y(t)|)}=4 t, t \in\right] 0,1[ \\
y(0)=0 \\
y(1)=1 \\
D^{0.2}\left(D^{\frac{1}{2}} y(0)\right)=0 \\
D^{0.2}\left(D^{0.2}\left(D^{\frac{1}{2}} y(0)\right)\right)=0 \\
D^{0.4}\left(D^{0.2}\left(D^{0.2}\left(D^{\frac{1}{2}} y(0)\right)\right)\right)=0 \\
D^{0.4}\left(D^{0.4}\left(D^{0.2}\left(D^{0.2}\left(D^{\frac{1}{2}} y(1)+\phi_{\frac{2}{5}, 0.2}(1) y(1)\right)\right)\right)\right)=0 \\
\phi_{\frac{2}{5}, 0.2}(t)=\frac{4}{10 t^{0.2}}
\end{array}\right.
$$

We have:

$$
\begin{aligned}
& f\left(t, x_{1}, x_{2}\right)=\frac{\sin x_{1}+\sin x_{2}}{100 e^{t^{2}+1}}, \quad g\left(t, x_{1}, x_{2}\right)=\frac{1}{48 e^{t}}\left(\cos x_{1}+\frac{\left|x_{2}\right|}{1+\left|x_{2}\right|}\right), \\
& h(t, x)=\frac{|x|}{\left(144 e^{t^{2}}\right)(1+|x|)}, \quad l(t)=4 t
\end{aligned}
$$

and

Also, we have

$$
\delta=\frac{1}{2}, \quad \rho=\frac{1}{7}
$$

$$
\left|f\left(t, x_{1}, x_{2}\right)\right| \leq \frac{1}{50}, \quad\left|g\left(t, x_{1}, x_{2}\right)\right| \leq \frac{1}{24}, \quad|h(t, x)| \leq \frac{1}{144}, \quad\|l\|_{\infty}=4,
$$

Since $f, g, h$ are continuous, then by Theorem 3.2, the problem presented in this example has at least one solution on $[0,1]$.

\section{Conclusion}

The aim of this paper was to introduce the more general singular fractional problem of Lane Emden type given in (8), by means of $n$ sequential Caputo derivatives. Using the well known Banach fixed point theorem, an existence and uniqueness result has been proved, then by application of Scahefer fixed point theorem, the existence of at least one solution for (8) has also been discussed. To justify the applicability of our main results, two examples have been studied in details.

Since in this field of interest it is important to increase ability of researchers for investigating differential equations and trying to find applicability of the studied problems in real word phenomena, in the paper we have discussed a Lane Emden type problem with a time singularity. However, for future developments, we think it will be more suitable to discuss the 
above problem by considering new nonlinearities with an arbitrary time singularity or with singularities in space variables. Another important physical point when dealing with the type of space singularities is to be concerned with positive solutions for the considered problem in order better to satisfy the needs of physicist researchers and to contribute with them in physical applications.

Acknowledgment. This paper is supported by La Direction Générale DGRSDT, Algiers, Algeria.

\section{References}

[1] M.A. Abdellaoui, Z. Dahmani, N. Bedjaoui, New Existence Results for a Coupled System on Nonlinear Differential Equations of Arbitrary Order, IJNAA, Vol. 6, No. 2 (2015).

[2] R.P. Agarwal, D. O'Regan, S. Staněk, Positive Solutions for Mixed Problems of Singular Fractional Differential Equations, Mathematische Nachrichten., Vol. 285, No. 1, (2012).

[3] A. Agila, D. Baleanu, R. Eid, B. Irfanoglu, Applications of the extended fractional Euler-Lagrange equations model to freely oscillating dynamical systems, Rom. J. Phys. 61(3-4), 350-359 (2016).

[4] R. Almeida, B.R.O. Bastos, M.T.T. Monteiro, Modeling some real phenomena by fractional differential equations, Math. Methods Appl. Sci. 39(16), 48464855 (2016).

[5] Y. Bahous, Z. Dahmani, A Lane Emden Type Problem Involving Caputo Derivative and Riemann-Liouville Integral,Indian Journal of Industrial and Applied Mathematics. Vol. 10, No. 1, (2019).

[6] Z. Bai , W. Sun, Existence and Multiplicity of Positive Solutions for Singular Fractional Boundary Value Problems, Computers \& Mathematics with Applications., Vol. 63, No. 9, (2012).

[7] D. Baleanu, H. Mohammadi, S. Rezapour, The existence of solutions for a nonlinear mixed problem of singular fractional differential equations, Adv. Differ. Equ. 2013, Article ID 359 (2013).

[8] Z. Bekkouche, Z. Dahmani, G. Zhang: Solutions and Stabilities for a 2D-Non Homogeneous Lane-Emden Fractional System, Int. J. Open Problems Compt. Math., Vol. 11, No. 2, (2018).

[9] A. Benzidane, Z. Dahmani, A class of nonlinear singular differential equations, Journal of Interdisciplinary Mathematics, Vol. 22, No. 6, (2019).

[10] R.C. Calleja, A.R. Humphries, B. Krauskopf, Resonance phenomena in a scalar delay differential equation with two state-dependent delays, SIAM J. Appl. Dyn. Syst. 16(3), 14741513 (2017).

[11] A. Carpinteri, F. Mainardi, Fractional Calculus in Continuum Mechanics, Springer, New York, NY (1997).

[12] Z. Dahmani, M.A. Abdellaoui, M. Houas: Coupled Systems of Fractional Integro-Diferential Equations Involving Several Functions, Theory and Applications of Mathematics . Computer Science 5 (1) (2015).

[13] Z. Dahmani, M.Z. Sarikaya, On a generalized Lane-Emden fractional differential system and its stability, Accepted on IASR-JARDCS of Dynamical and Control Systems. Accepted (2016).

[14] Z. Dahmani, A. Taeib, N. Bedjaoui, Solvability and Stability for Nonlinear Fractional Integro-Differential Systems of High Fractional Orders, Facta Nis Ser. Math. Inform., Vol. 31, No. 3, (2016).

[15] R. Emden, Gaskugeln, Teubner, Leipzig and Berlin, (1907).

[16] Y. Gouari, Z. Dahmani, M.Z. Sarikaya, A non local multi-point singular fractional integro-differential problem of lane-emden type. Math Meth Appl Sci.(2020).

[17] M. Houas, Z. Dahmani, New fractional results for a boundary value Problem with Caputo Derivative, Int. J. open Problems Compt. Math., 6(2) (2013).

[18] R.W. Ibrahim, Existence of Nonlinear Lane-Emden Equation of Fractional Order, Miskolc Mathematical Notes, Vol. 13, No. 1, (2012).

[19] R.W. Ibrahim, Stability of A Fractional Differential Equation, International Journal of Mathematical, Computational, Physical and Quantum Engineering., Vol. 7, No. 3, (2013).

[20] A.A. Kilbas, H.M. Srivastava, J.J. Trujillo, Theory and Applications of Fractional Differential Equations, Elsevier B.V., Amsterdam, The Netherlands, (2006). 
[21] D. Kumar, J. Singh, S. Dutt Purohit, R. Swroop, A hybrid analytical algorithm for nonlinear fractional wave-like equations, Mathematical Modelling of Natural Phenomena 14, 304(2019).

[22] C. Li , S. Sarwar, Existence and Continuation of Solutions for Caputo Type Fractional Differential Equations, Electron. J.Differential Equations., Vol. 2016, No. 207, (2016).

[23] S.M. Mechee, N. Senu, Numerical Study of Fractional Differential Equations of Lane-Emden Type by Method of Collocation, Applied Mathematics, 3, (2012).

[24] K.S. Miller, B. Ross, An Introduction to the Fractional Calculus and Fractional Differential Equations, Wiley, New York., (1993).

[25] A. Mohammadi, N. Aghazadeh, S. Rezapour, Haar wavelet collocation method for solving singular and nonlinear fractional time-dependent Emden-Fowler equations with initial and boundary conditions, Math. Sci., (2019).

[26] S.A. Okunuga, J.O. Ehigie, A.B. Sofoluwe, Treatment of Lane-Emden Type Equations via Second Derivative Backward Differentiation Formula Using Boundary Value Technique, Proceedings of the World Congress on Engineering., Vol. IWCE, (2012).

[27] J. Serrin , H. Zou, Existence of Positive Solutions of Lane-Emden Systems Atti Del Sem, Mat. Fis. Univ. Modena., 46 (Suppl), (1998).

[28] M. Yigider, K. Tabatabaei, E. elik, The numerical method for solving differential equations of Lane-Emden type by Padé approximation. Discrete Dyn. Nat. Soc. (2011). 